\title{
La politique des partis en Grande-Bretagne pendant la Révolution française
}

Essai de parcours bibliographique

James M. A. Inglis

\section{(Q) OpenEdition \\ Journals}

\section{Édition électronique}

URL : https://journals.openedition.org/ahrf/10202

DOI : 10.4000/ahrf.10202

ISSN : 1952-403X

Éditeur :

Armand Colin, Société des études robespierristes

Édition imprimée

Date de publication : 15 mars 2006

Pagination : 195-198

ISSN : 0003-4436

\section{Référence électronique}

James M. A. Inglis, « La politique des partis en Grande-Bretagne pendant la Révolution française », Annales historiques de la Révolution française [En ligne], 343 | janvier-mars 2006, mis en ligne le 01 mars 2009, consulté le 22 avril 2022. URL : http://journals.openedition.org/ahrf/10202 ; DOI : https:// doi.org/10.4000/ahrf.10202

Ce document a été généré automatiquement le 22 avril 2022.

Tous droits réservés 


\section{La politique des partis en Grande- Bretagne pendant la Révolution française}

Essai de parcours bibliographique

James M. A. Inglis

Des ouvrages généraux

Harry T. Dickinson (ed.), Britain and the French Revolution 1789-1815, Basingstoke, Macmillan, 1989.

Jennifer Mori, Britain in the Age of the French Revolution, Harlow, Pearson Education, 2000.

Clive Emsley, Britain and the French Revolution, Harlow, Pearson Education, 2000.

4 Au cours de la Révolution française, la Chambre des Communes bénéficia de la présence en son sein de quelques-unes des plus illustres figures politiques de l'histoire britannique moderne, parmi lesquelles William Pitt «le Jeune » et Charles James Fox. Au cours des années 1790 et des quinze premières années du dix-neuvième siècle, la Grande-Bretagne fut directement impliquée dans la lutte contre la France révolutionnaire. C'est durant ces années que se déroulèrent d'importantes évolutions politiques, avec le déclin de l'influence monarchique et la montée du système bipartite, ainsi que des changements économiques et sociaux liés à la Révolution industrielle. Les historiens de la période cherchent à expliquer ces développements et à évaluer l'impact des idées révolutionnaires françaises et de ces nombreuses années de guerre.

La collection d'articles éditée par H. T. Dickinson examine l'impact de la Révolution française et des guerres révolutionnaires sur le radicalisme et le loyalisme, sur l'Irlande, sur la littérature, sur les évolutions économiques et sociales et sur la conduite de la guerre et de la diplomatie. Des articles importants portent sur les high politics ${ }^{1}$, explorant l'impact des événements en France sur Pitt et le parti gouvernemental, sur Fox et l'opposition parlementaire, ou encore sur les finances publiques, soit des enjeux vitaux de l'effort de guerre britannique ${ }^{2}$. L'ouvrage de Jennifer Mori est un précieux travail de synthèse, appuyé sur une large lecture des sources imprimées, dont la liste 
est fort utilement reproduite en fin d'ouvrage. L'auteur n'essaie pas d'offrir un récit chronologique des années 1785-1820, mais propose sept chapitres analytiques examinant les politiques des partis, l'opposition idéologique entre les radicaux et les loyalistes, les changements sociaux et économiques, et le financement et la conduite des longues guerres contre la France. Le court livre de Clive Emsley est avant tout un manuel, qui offre une discussion utile sur trois thèmes: l'impact de la Révolution française sur les luttes idéologiques et politiques internes à la Grande-Bretagne et l'impact des guerres révolutionnaires sur la société britannique. Ces chapitres sont suivis par 29 documents choisis parmi les sources primaires.

Du système politique

6 John W. Derry, Politics in the Age of Fox, Pitt and Liverpool, Basingstoke, Palgrave, 2001.

7 Frank O'Gorman, The Emergence of the British Two-Party System 1760-1832, London, Edward Arnold, 1982.

8 Frank O'Gorman, Voters, Patrons, and Parties: The Unreformed Electoral System of Hanoverian England 1734-1832, Oxford, Clarendon Press, 1989.

R.G. Thorne (ed.), The House of Commons 1790-1820, 5 vols., London, Secker and Warburg, 1986.

10 En 1789, William Pitt « le Jeune » était Premier Ministre : il domina le paysage politique pendant toute la période révolutionnaire jusqu'à sa mort en 1806. Au départ, il accorda assez peu d'intérêt à la Révolution française, mais c'est lui qui conduisit le pays à la guerre contre la France afin de préserver l'équilibre du pouvoir européen. En 1794, l'aile conservatrice de l'opposition parlementaire, menée par le duc de Portland, rejoignit le gouvernement afin de créer une union nationale contre la France. Charles James Fox resta dans l'opposition et dirigea un parti-croupion, démoralisé à la fin des années 1790, luttant pour mettre en place une alternative valable à Pitt. En 1801, devant l'impossibilité de convaincre le roi George III d'accepter l'émancipation des Catholiques, Pitt démissionna. Son allié, Henry Addington, tenta alors de former une administration pittite, mais Pitt et nombre de ses alliés les plus importants, notamment Dundas et Grenville, refusèrent d'être au service d'Addington. Ce dernier signa la paix d'Amiens avec la France en 1802 : elle ne produisit qu'une brève trêve et le ministre fut gravement affaibli par la reprise de la guerre contre la France napoléonienne en 1803. Pitt fut ensuite invité à former un autre gouvernement en 1804, mais William Grenville, son principal allié dans les années 1790 , refusa un ministère lorsque le roi s'opposa de nouveau à l'octroi de toute concession aux catholiques. À la mort de Pitt en 1806, Grenville mena une coalition gouvernementale, "le ministère de tous les talents ", qui incluait Fox comme secrétaire d'État des Affaires étrangères. Ce cabinet ne survécut pas longtemps, sapé par la mort de Fox en 1806, la démission d'Addington en 1807, et le refus persistant du roi d'accorder des concessions politiques à ses sujets catholiques.

11 Le duc de Portland devint Premier Ministre en 1807 et chercha à ramener les Pittites au pouvoir. Âgé et malade, il ne parvint qu'à grand peine à contrôler ses collègues de cabinet et fut affaibli par les victoires françaises à l'étranger. À la retraite de Portland en 1809, c'est Spencer Perceval qui devint Premier Ministre. Il réussit à faire revenir Addington et Castlereagh dans son cabinet, mais échoua à y faire entrer Canning et Grenville. Perceval fut assassiné par un faible d'esprit en 1812, un nouveau gouvernement fut difficilement formé sous la direction du duc de Liverpool. Progressivement, le nouveau Premier Ministre réussit à établir son autorité sur un cabinet frondeur et parvint à réunifier et à renforcer le parti pittite des années 1790, 
bénéficiant du retournement de conjoncture dans la longue guerre contre la France napoléonienne. Liverpool fut donc un Premier Ministre qui collectionna les succès jusqu'à sa retraite, due à la maladie, en 1827.

12 La courte mais utile synthèse de Franck O'Gorman, publiée en 1982, analyse le retour graduel du système bipartite entre 1760 et 1832 . Le premier chapitre couvre le renouveau d'un parti Whig, hostile au patronage et aux prérogatives de la couronne, privilégiant en revanche une attitude plus libérale envers la liberté religieuse et la réforme politique. Le chapitre suivant retrace le développement d'un parti Tory, favorable à la conservation des institutions traditionnelles de l'Église et de l'État face aux menaces incarnées par les révolutionnaires français et les radicaux britanniques. Le troisième chapitre aborde la question de l'évolution du système bipartite au parlement et dans les circonscriptions électorales au début du XIX siècle et le dernier chapitre analyse l'étendue de l'activité partisane lors du premier gouvernement de Liverpool. Le petit ouvrage de John Derry couvre à peu près la même période et livre un récit des principaux événements de la politique parlementaire entre l'accession de Pitt au pouvoir en 1783 et la démission de Liverpool en 1827. Ce court survol est un travail de synthèse, mais Derry a passé de nombreuses années à travailler sur ce thème.

Le travail magistral d'O'Gorman sur les élections examine les types de circonscriptions dans le pays, le profil des candidats, la façon de gagner un siège au parlement et les facteurs qui affectent le comportement des votants. Les cinq volumes édités par R. G. Thorne fournissent une masse d'informations détaillées sur les députés et leurs circonscriptions entre 1790 et 1820 . Le premier volume livre un bon aperçu du système électoral et les résultats de chacune des élections générales pendant ces trois premières décades. Il inclut une liste des candidats malheureux et des élections contestées. Le second volume donne un compte rendu précis de l'activité électorale dans chaque circonscription parlementaire du pays. Enfin, les trois derniers volumes fournissent un état de l'activité politique de chacun des députés qui furent membres du parlement à un moment ou à un autre de la période. C'est donc une source importante contenant un véritable trésor d'informations.

De la monarchie University Press, 1988. G.M. Ditchfield, George III : An Essay in Monarchy, Basingstoke, Palgrave Macmillan, 2002.

Christopher Hibbert, George III : A Personal History, London, Viking, 1998.

Saul David, Prince of Pleasure: The Prince of Wales and the Making of the Regency, London, Little, Brown and Company, 1998.

Steven Parissien, George IV : The Grand Entertainment, London, John Murray, 2001.

E.A. Smith, George IV, New Haven, Yale University Press, 1999.

20 En 1789, George III, après un règne de 30 ans, était un politicien très expérimenté. Il avait établi des relations de travail cordiales avec son Premier Ministre, William Pitt, et était déterminé à maintenir à l'écart du jeu du pouvoir Charles James Fox, ami intime de son fils aîné et héritier, le prince de Galles. Scandalisé par les débuts de la Révolution et horrifié par l'exécution de Louis XVI, il se réjouit du déclenchement de la guerre avec la France. Malgré les défaites, il encouragea sans jamais fléchir l'effort de guerre. Toutefois, en 1801, le monarque affronta son Premier Ministre sur le problème de l'émancipation catholique, préférant encore la démission de Pitt plutôt que de 
transiger sur la question. C'est cette même réticence envers toute concession accordée aux catholiques qui mena à la démission de Lord Grenville, alors Premier Ministre, en 1806 , et à la chute du « ministère de tous les talents » en 1807.

21 La mauvaise santé du roi, et finalement sa folie, eut un effet important sur l'évolution politique, déstabilisant de nombreuses administrations et contribuant au final à fragiliser l'influence de la couronne. Pitt, comme d'autres, n'était guère enthousiaste à l'idée de privilégier une politique qui aurait pu entraîner une rechute et une nouvelle crise politique. Le roi eut un premier accès de folie en 1801, lors de la crise provoquée par l'émancipation catholique. Lorsqu'Addington fut attaqué au Parlement, en 1804, la maladie s'aggrava. La mauvaise santé du roi souleva bientôt la perspective d'une régence et la peur que le prince de Galles ne remplace les ministres de son père par Fox et d'autres politiciens issus de l'opposition. En 1810, George III sombra définitivement dans la folie: son fils aîné fut nommé régent avec des pouvoirs restreints l'année suivante, puis prince Régent doté des pleins pouvoirs en 1812. Le prince de Galles fut lui-même une source d'instabilité politique bien avant d'avoir pu exercer un quelconque pouvoir. Pendant des années, il mena une vie dissolue, frayant avec Fox, Sheridan et d'autres leaders de l'opposition. En 1788-1789, la perspective de le voir devenir régent produisit d'ailleurs une crise majeure, qui ne s'acheva que lorsque George eut recouvré sa santé mentale. Lorsqu'il devint finalement prince Régent, on s'attendit à ce qu'il révoque Perceval et amène ses vieux amis de l'opposition au gouvernement. De fait, il conserva les ministres nommés par son père, remplaçant même Perceval, après l'assassinat de ce dernier, par un ministère dirigé par Lord Liverpool et dominé par des Pittites. Les relations avec Liverpool ne furent jamais très paisibles et débouchèrent même sur une crise lorsque George succéda à son père sur le trône, cherchant en vain à divorcer, ayant été séparé de son épouse depuis de nombreuses années.

Marilyn Morris a mené des recherches sur les répercussions de la Révolution sur la monarchie britannique. Sa monographie informée commence par une analyse des fondements traditionnels de l'autorité royale à la fin du XVIII ${ }^{e}$ siècle, avant d'examiner l'impact de la Révolution sur la défense de l'idée monarchique en Grande-Bretagne. On ne dispose toujours pas de biographie réellement satisfaisante sur George III. La biographie écrite par Stanley Ayling en 1972 est un récit utile des événements, mais une analyse approfondie du contexte politique dans lequel s'inscrirait le rôle du roi lui fait défaut. La biographie écrite par John Brooke la même année témoigne d'une sensibilité plus fine à la complexité politique de la période, mais reste indéniablement trop légère sur la période d'après 1780 . Christopher Hibbert a, quant à lui, donné une histoire personnelle du roi, s'appuyant sur des recherches importantes: le livre convainc lorsqu'il traite des relations du roi avec sa femme et ses nombreux enfants, mais n'essaie pas vraiment d'explorer le rôle politique du roi. Le George III de G. M. Ditchfield n'est pas une biographie traditionnelle, mais reste l'étude la plus utile dont nous disposions sur ce monarque, tout particulièrement sur la fin de son règne. Si l'ouvrage est bien documenté, son objet n'est pas de faire une étude chronologique de la vie privée et publique de George. C'est fondamentalement une étude par thèmes, qui examine des sujets comme l'image du roi, telle que l'histoire l'a recomposée, l'implication du monarque dans les affaires politiques européennes, sa contribution à la politique impériale du gouvernement, et ses opinions religieuses, en lien avec sa participation active dans les affaires de l'Église. D'après Ditchfield, le roi est donc un 
acteur central de la vie politique britannique jusqu'à ce qu'il sombre définitivement dans la folie en 1810.

Le Prince of Pleasure de Saul David relève de l'étude biographique, l'auteur s'étant penché sur la jeunesse de George IV jusqu'à son accession au trône en 1820. S'il s'appuie sur des recherches originales, c'est un ouvrage de vulgarisation, centré sur la vie privée plutôt que sur la carrière publique du prince, même après que ce dernier fut devenu Régent. Cet ouvrage ne remplace pas la biographie antérieure, bien plus détaillée et plus complète que Christopher Hibbert a consacrée au même homme (en deux volumes séparés en 1972-1973, puis dans un volume unique en 1976). La biographie de E. A. Smith est un ouvrage académique plus ambitieux, qui rompt avec les précédents en analysant en profondeur les relations du roi avec son père George III, ses frères et sœurs, ou encore son mécénat culturel et artistique et son rôle politique. S'il accorde au prince Régent la place qui lui est due dans le soutien à la guerre contre la France napoléonienne, il va sans doute trop loin sur d'autres questions. Il cherche ainsi à laver la réputation exécrable du Régent, ou prétend qu'il est partiellement à l'origine de la réduction du rôle public de la monarchie britannique, affaiblissement, d'ailleurs, qu'il aurait accepté. Enfin, le livre de Steven Parissien est une étude thématique, et non une biographie traditionnelle. S'il s'intéresse à la vie privée agitée du prince et livre quelques observations sur son rôle politique, la majeure partie de l'étude est consacrée au patronage des architectes par le roi, à son goût pour les beaux-arts et son intérêt pour la mode, particulièrement les uniformes.

De la biographie

On consultera aussi avec profit les notices du New Dictionary of National Biography, Oxford, Oxford University Press, 2004.

- Henry Addington, 1st Viscount Sidmouth (1757-1844)

Philip Ziegler, Addington : A Life of Henry Addington, First Viscount Sidmouth, London, Collins, 1965.

Charles John Fedorak, Henry Addington, Prime Minister, 1801-1804 : Peace, War, and

Parliamentary Politics, Akron, GA, University of Akron Press, 2002.

En 1783, Henry Addington entre au parlement. Se liant d'amitié avec Pitt, il est élu Speaker de la Chambre des Communes en 1789. En 1801, il succède à Pitt comme Premier Ministre, négocie une paix de courte durée avec Napoléon en 1802, mais reprit les hostilités en 1803. Cette guerre affaiblit sa majorité et encouragea de nombreux députés à se tourner vers Pitt pour sauver le pays. Addington fut finalement obligé de démissionner en 1804 et fut remplacé par Pitt. Grâce à une réconciliation de courte durée avec ce dernier, il fut anobli en 1805. Il servit ensuite dans le ministère de « tous les talents » en 1806-1807, puis revint, avec ses amis, dans le camp de Pitt en 1812. Liverpool fit rapidement de lui son ministre de l'Intérieur, une position qu'il conserva jusqu'à sa retraite politique en 1824. Il est considéré comme un ministre de l'Intérieur répressif, qui réagit avec intransigeance aux désordres industriels et aux activités radicales pendant ses années passées au gouvernement.

Philip Ziegler a écrit la seule biographie académique sur Addington, et propose une recherche détaillée et approfondie de sa longue carrière politique. Son étude souffre cependant d'avoir été rédigée avant la publication de très nombreuses études sur l'histoire politique de cette époque. Le livre de Fedorak est, quant à lui, plus à jour et s'appuie sur une étude impressionnante des sources disponibles. C'est un bon compte rendu du contexte politique et il démontre de façon convaincante qu'Addington était 
bien plus qu'un Premier Ministre de second ordre. Néanmoins, ce travail ne couvre qu'une faible partie de la longue et diverse carrière d'Addington.

- Edmund Burke (1730-97)

Stanley Ayling, Edmund Burke : His Life and Opinions, London, Cassell, 1988.

J.C.D. Clark (ed.), Edmund Burke: Reflections on the Revolution in France: A Critical Edition,

Stanford, CA, Stanford University Press, 2001.

F.P. Lock, Burke's Reflections on the Revolution in France, London, George Allen \& Unwin, 1985.

F.P. Lock, Edmund Burke : Vol. I : 1730-1784, Oxford, Clarendon Press, 1998.

Conor Cruise O'Brien, The Great Melody : A Thematic Biography, London, Sinclair-Stevenson, 1992. Sa célébrité trouve son origine dans ses discours et écrits, portant sur la politique intérieure, la crise américaine, la politique indienne et irlandaise de la Grande-Bretagne, et plus tard sur la Révolution française et les guerres révolutionnaires. Proche allié de Fox, et opposant de Pitt durant les années 1780, sa carrière connut un tournant spectaculaire à la suite du déclenchement de la Révolution française. Dès novembre 1790, Burke publia ses célèbres Réflexions sur la Révolution de France, critique féroce des principes révolutionnaires français et dénonciation des radicaux britanniques. Conséquences directes, la rupture douloureuse avec Fox et la décision de Burke de soutenir Pitt dans son hostilité envers l'évolution politique française. Il devint un partisan fervent de la guerre contre la France, une guerre qu'il voulait mener contre les principes révolutionnaires, et non contre le peuple français. Il croyait, par conséquent, qu'aucune paix n'était possible en Europe avant que les principes révolutionnaires ne soient renversés.

Il n'est pas étonnant que les actes, les discours et les écrits de Burke aient été étudiés par de nombreux historiens, spécialistes d'histoire politique ou d'histoire des idées politiques. Pourtant, il n'existe pas de biographie de Burke réellement satisfaisante. La première biographie à paraître après la publication de la correspondance de Burke est celle de Stanley Ayling : ce n'est pas un travail de recherche très approfondi. S'il décrit de façon utile la carrière politique de Burke, il ne rend pas justice à la complexité et à la sophistication de ses idées politiques. La biographie de Conor Cruise O'Brien est, quant à elle, thématique. Le livre est très intéressant lorsqu'il aborde la vie personnelle de Burke et est même excellent lorsqu'il traite de son identité irlandaise, mais s'essouffle dans les derniers chapitres. Les passages les plus faibles concernent la réponse burkienne à la Révolution. F. P. Lock pourrait bien être en train d'écrire la meilleure biographie de Burke. Le premier volume publié, qui traite de la carrière de Burke jusqu'à la fin de la guerre d'indépendance américaine, est un excellent travail de recherche, combinant le récit et l'analyse, étudiant aussi bien les actions de Burke que ses discours et écrits. Concernant Burke et la Révolution française, les historiens qui s'intéressent à Burke verront avec intérêt que Lock a également écrit une étude précieuse sur l'apparition des Réflexions. Il y explore le contexte, l'élaboration, la signification, la rhétorique et la réception de cet ouvrage extrêmement influent. On dispose de nombreuses éditions des Réflexions, mais la meilleure est l'édition critique de J.C.D. Clark, qui propose une critique remarquable sur cet ouvrage fondamental.

- Henry Dundas, 1st Viscount Melville (1742-1811)

- Robert Saunders Dundas, 2nd Viscount Melville (1771-1851)

Michael Fry, The Dundas Despotism, Edinburgh, Edinburgh University Press, 1992. 
Henry Dundas géra les affaires écossaises tout au long des deux premiers gouvernements Pitt. Allié proche de celui-ci, il fut Trésorier de la Marine de 1783 à 1800, ministre de l'Intérieur de 1791 à 1794, et ministre de la Guerre de 1794 à 1801 . Il devint Premier Lord de l'Amirauté en 1805, mais sa carrière cessa brutalement lorsque l'on découvrit la mauvaise gestion des affaires dans l'administration navale. Même s'il échappa personnellement à la condamnation pour corruption, il fut poussé à la démission. Son fils, Robert Dundas, entra au Parlement en 1796, puis devint ministre en 1807, en tant que Président du Board of Control. Au gouvernement, il prit la suite de son père comme chargé des affaires écossaises, une place qu'il occupa jusqu'en 1827. Il servit également comme Premier Lord de l'Amirauté de 1812 à 1827.

L'ouvrage de Michael Fry est la première étude contemporaine de ces deux politiciens. Il fait bon usage des sources, mais reste malgré tout très journalistique dans son style. Tout en reconnaissant l'influence exercée par les deux Dundas sur les affaires écossaises, il nuance la vision traditionnelle d'un régime corrompu et tyrannique.

- John Scott, 1st Earl of Eldon

R.A. Melikan, John Scott, Lord Eldon 1751-1838 : The Duty of Loyalty, Cambridge, Cambridge

University Press, 1999.

31 John Scott, qui devint plus tard Lord Eldon, fut l'un des principaux juristes de son temps et un ardent sympathisant de Pitt dans les années 1790. Il détint le poste de Procureur général [Attorney General] de 1793 à 1799 et joua un rôle majeur dans les efforts gouvernementaux pour condamner les radicaux. Il fut nommé Lord Chancellor en 1807, ne collabora pas au ministère "de tous les talents", puis redevint Lord Chancellor et occupa ce poste jusqu'à sa démission en 1827. Tout au long de ces années, il s'opposa constamment à toute réforme politique. La biographie de Melikan est la seule biographie récente sur ce politicien conservateur de premier plan. Elle s'appuie sur une étude exhaustive des sources, et rend justice à la double carrière judiciaire et politique d'Eldon.

- Charles James Fox (1749-1806)

Stanley Ayling, Fox: The Life of Charles James Fox, London, John Murray, 1991.

L.G. Mitchell, Charles James Fox, London, 1992.

Charles James Fox fut le principal opposant politique de Pitt, de l'accession de ce dernier au poste de Premier Ministre en 1783, jusqu'à sa mort en 1806. Fox accueillit chaleureusement le déclenchement de la Révolution française, admirant la nouvelle constitution de 1791, il s'opposa à la guerre contre la France tout en critiquant la politique répressive menée par Pitt contre les réformateurs britanniques. Cette attitude finit par lui aliéner nombre de ses alliés plus conservateurs. Burke rompit ainsi avec lui en 1792, tandis que le duc de Portland conduisit une bonne partie des Whigs d'opposition à s'allier avec Pitt en 1794. Fox persista à rendre Pitt responsable de la guerre et de l'échec à faire la paix avec la France. Il abandonna pratiquement toute activité politique à la fin des années 1790 , mais se réjouit de la paix d'Amiens en 1802, son arrivée à Paris donnant lieu à des célébrations. À partir de 1804 s'engagea son alliance avec Grenville, qui déboucha sur une charge suprême en 1806, lorsqu'il devint ministre des Affaires étrangères dans le ministère "de tous les talents ». Il échoua cependant à négocier la paix avec Napoléon, mais défendit l'abolition du commerce des esclaves avant sa mort, peu de temps après celle de son grand rival Pitt. 

imprimées que Trevelyan n'a pu consulter. En particulier, l'ouvrage aborde en détail la longue période passée par Grey dans l'opposition, ainsi que ses quatre années comme Premier Ministre au début des années 1830. Quoique bien plus court et moins probant, le livre de Derry s'appuie aussi sur des sources originales et tente de couvrir l'ensemble de la carrière publique de Grey. En revanche, la vie privée de Grey, pourtant très 
intéressante, est largement ignorée. Quatre des six chapitres sont centrés sur la carrière de Grey entre 1789 et 1815, mais de fait, les passages sur la guerre contre la France concernent davantage Pitt que Grey.

- William Pitt the Younger (1759-1806)

John Ehrman, The Younger Pitt : The Consuming Struggle, London, Constable, 1996.

Jennifer Mori, William Pitt and the French Revolution 1785-1795, Edinburgh, Keele University

Press, 1997.

Eric J. Evans, William Pitt the Younger, London, Routledge, 1999.

Michael Duffy, The Younger Pitt, Harlow, Longman, 2000.

Michael J. Turner, Pitt the Younger : A Life, London, Hambledon and London, 2003.

William Hague, William Pitt the Younger, London, Harper Collins, 2004.

À seulement 24 ans, fin 1783, Pitt fut choisi comme Premier Ministre par George III. Il détint ce poste jusqu'à sa démission en 1801, d'abord en raison du refus royal d'accorder l'émancipation aux catholiques, ensuite à cause du surmenage accumulé par ses longues années au pouvoir et par l'épuisante et coûteuse guerre contre la France révolutionnaire. $\mathrm{Si}$, à l'origine, Pitt n'était pas hostile à la Révolution française, il s'inquiéta dès lors que les ambitions françaises s'étendirent aux Provinces-Unies. Lorsque la guerre éclata, Pitt consacra d'immenses efforts à la constitution d'une coalition européenne efficace contre la France, et à la recherche de financements pour l'effort de guerre. Cependant, à la différence de son père, ce ne fut jamais un grand stratège militaire, et il ne sut jamais trancher entre une guerre terrestre, sur le continent, et une guerre navale et coloniale outre-mer. Dans les îles britanniques, il mit en place une série de mesures répressives pour défaire les radicaux, vainquant également la rébellion irlandaise en 1798, et mena à son terme l'union entre la GrandeBretagne et l'Irlande avant de quitter son poste. Il vécut assez longtemps pour apprendre la victoire de Nelson à Trafalgar et la stupéfiante victoire de Napoléon à Austerlitz, mais mourut en 1806 à seulement 46 ans.

C'est en 1996 que John Ehrman a mis la dernière touche à son imposante et remarquable biographie de Pitt, en trois volumes. Le premier volume, The Years of Acclaim, fut publié en 1969, et le second, The Reluctant Transition, en 1983. Au total, c'est l'une des meilleures biographies jamais écrites sur un politicien britannique d'envergure. Ehrman passa de nombreuses années à penser cette œuvre, consultant toutes les sources sur le sujet. Sa biographie couvre tous les aspects de la carrière de Pitt, dans les moindres détails, et ses conclusions emportent l'adhésion. Afin de faire honneur à la considérable réussite d'Ehrman, le journal History a publié (volume 83, avril 1998), six essais sur différents aspects de la carrière de Pitt. Le livre de Jennifer Mori, version remaniée de sa thèse de doctorat, est quant à lui d'une portée bien plus limitée, mais c'est un travail de qualité sur l'attitude de Pitt envers la France entre 1785 et 1795. Cette historienne examine comment sa politique, sur le front intérieur et outre-mer, fut guidée par sa réaction à la Révolution française. Enfin, Eric Evans propose une courte mais utile introduction à la carrière de Pitt, assez substantielle pour ce qui touche aux relations de Pitt avec George III, la guerre avec la France et l'union avec l'Irlande.

40 Depuis que Ehrman a mis le point final à sa biographie magistrale, trois nouvelles biographies de qualité sont apparues. Toutes trois s'appuient nettement sur le travail d'Ehrman, sans pour cela n'en être que des produits dérivés. En effet, l'ouvrage de Duffy est avant tout une étude thématique, mettant en valeur les aspects centraux de la 
carrière de Pitt. Ce livre aborde aussi les relations du jeune premier ministre avec le roi, ses collègues ministériels et ses partisans au parlement. D'autres chapitres traitent de son rôle dominant dans la stratégie politique de la Grande-Bretagne, le contrôle qu'il sut établir sur la Chambre des Communes, ses appels à la nation tout entière et son rôle dans la guerre contre la France. La biographie écrite par Turner est un récit chronologique plus traditionnel. Sans surprises ni faiblesses majeures, l'étude est solide et sensée. William Hague, politicien de métier, a écrit une biographie encore plus longue. S'il s'appuie largement sur Ehrman, il parvient à ressusciter le Premier Ministre et est particulièrement intéressant sur Pitt l'orateur et les grands débats parlementaires dans lesquels s'impliqua ce dernier.

- William Henry Cavendish Bentinck, 3rd Duke of Portland (1738-1809)

David Wilkinson, The Duke of Portland : Politics and Party in the Age of George III, Basingstoke,

Palgrave Macmillan, 2003.

41 Portland fut très brièvement Premier Ministre en 1783, puis passa une décennie dans l'opposition à Pitt, en tant que proche allié de Charles James Fox. De plus en plus convaincu par la vision burkienne de la Révolution française, il rompit finalement et à contrecœur avec Fox, menant la majorité de l'opposition à former une coalition avec Pitt en 1794. En qualité de ministre de l'Intérieur [home secretary], il mit en œuvre les mesures répressives du gouvernement contre les radicaux. C'est à ce même titre qu'il fut impliqué dans la politique britannique en Irlande, dont il souhaitait ardemment l'union avec la Grande-Bretagne. Démissionnant en compagnie de Pitt en 1801, il ne revint au cabinet qu'en 1807, lorsqu'il devint un Premier Ministre «de paille » d'une administration pittite. Il lutta ainsi vainement pour conserver la pluralité des voix dans son gouvernement et ne fit pas beaucoup de progrès dans la guerre contre la France. Sa mauvaise santé le conduisit à se retirer de la vie politique peu avant de mourir, en 1809.

L'étude de David Wilkinson, qui s'appuie sur sa thèse de doctorat, est la seule biographie moderne sur cet homme politique d'envergure. Bien que fondé sur des recherches considérables, c'est un travail relativement court. L'auteur ne cherche pas à appréhender tous les aspects de la vie privée et publique de Portland. Ce livre est particulièrement intéressant lorsqu'il aborde la rupture avec Fox, le soutien apporté par Portland aux politiques répressives de Pitt, son utilisation des services secrets, ou encore l'éradication de la rébellion irlandaise.

- Richard Brinsley Sheridan

Linda Kelly, Richard Brinsley Sheridan : A Life, London, Sinclair-Stevenson, 1997.

Fintan O'Toole, A Traitor's Kiss : The Life of Richard Brinsley Sheridan, London, Granta Books, 1997.

On connaît généralement Sheridan en tant que dramaturge, bien qu'il ait été un politicien important pendant de nombreuses années. Né à Dublin, fils de comédien, il épousa une chanteuse célèbre, écrivit quelques-unes des plus belles pièces comiques de la fin du XVIII e siècle - toujours très populaires aujourd'hui - et fut même le propriétaire et l'administrateur du fameux Drury Lane Theater. Vivant toujours largement au-dessus de ses moyens, il parvint à entrer au parlement en 1780 et entama une longue carrière politique. Pendant de nombreuses années, ce fut un allié de Fox et un partisan du prince de Galles, et donc un opposant à la politique intérieure et extérieure de Pitt. Orateur talentueux, Sheridan fut impliqué dans l'impeachment de Warren Hastings, défendit la Révolution française et s'opposa aux politiques répressives de Pitt en Grande-Bretagne. La politique extérieure de Pitt fut également 
l'objet de ses critiques, en particulier la guerre contre la France, et Sheridan défendit même les United Irishmen dans les années 1798-99. En 1801-1802, il poussa à la paix avec la France, tout en s'opposant à l'Acte d'Union avec l'Irlande. Au sein du Ministère « de tous les talents ", Sheridan occupa le poste de Trésorier de la Marine. De plus en plus critique envers Napoléon, considéré comme versatile, il agit cependant toujours en faveur de l'émancipation catholique et afficha un intérêt continuel pour les affaires irlandaises.

La biographie publiée par Linda Kelly est assez complète quant à la vie privée et à l'œuvre théâtrale de Sheridan, mais ne rend pas justice à sa carrière politique. Survolant le contexte politique, elle n'essaie pas de voir précisément les enjeux dans lesquels ce politicien fut impliqué et le rôle exact qu'il y a joué. Fintan O'Toole, journaliste et critique théâtral en Irlande, rend mieux compte de la vie privée remarquable de Sheridan, de sa carrière artistique et de la production théâtrale de l'époque. Les choix politiques de Sheridan sont abordés, ainsi que la rhétorique de ses discours, mais cet auteur surévalue l'ampleur des réseaux irlandais de Sheridan, et son implication dans le radicalisme irlandais. Bienveillante, presque trop, envers son sujet, cette biographie est vivante et distrayante, mais trop superficielle en termes académiques. La carrière politique de Sheridan attend toujours son historien.

\section{NOTES}

1.Les historiens anglo-saxons distinguent ainsi (high politics) l'espace du pouvoir et des forces parlementaires, de celui de l'expression et du combat politique dans les milieux populaires, les meetings, la rue (popular politics), etc.

2.Frank O'Gorman, Jown Derry and Francois Crouzet.

\section{AUTEUR}

JAMES M. A. INGLIS

University of Edinburgh

School of History

Edinburgh, EH8 9JY, Écosse, Royaume-Uni

s.9453648@sms.ed.ac.uk 